\title{
Detection of different shapes and materials by glasses for blind and visually impaired
}

\author{
Urban Košale \\ Faculty of Electrical \\ Engineering and Computer \\ Science \\ University of Maribor \\ Maribor, Slovenia \\ urban.kosale@student.um.si
}

\author{
Pia Žnidaršič \\ Faculty of Electrical \\ Engineering and Computer \\ Science \\ University of Maribor \\ Maribor, Slovenia \\ pia.znidarsic@student.um.si
}

\author{
Kristjan Stopar \\ Faculty of Electrical \\ Engineering and Computer \\ Science \\ University of Maribor \\ Maribor, Slovenia \\ kristjan.stopar@student.um.si
}

\begin{abstract}
Glasses for blind and visually Impaired were built to help blind and visually impaired to navigate around diverse range of obstacles.

In this study, we tested how well the glasses detect different everyday shapes and materials. The results are crucial for further development, because this device has to able to detect wide variety of shapes and materials in order to be safe and reliable for everyday usage.
\end{abstract}

For this purpose, we set glasses on a stationary stand and pointed them directly into the obstacle centre. Obstacles made of different materials were attached on a moving stand. The results showed that the sensors discriminate the shapes at the distances between 30 and $90 \mathrm{~cm}$ from the glasses. At the distance of $60 \mathrm{~cm}$ the triangle was successfully discriminated from circle and rectangle, whereas the latter two were not easy to discriminate. The second experiment showed that plexi glass and glass present a substantial detection challenge. On the other hand, aluminium foil, white paper and micro polyester are easily detected.

\section{Keywords}

glasses for the blind and visually imapred, detection, sensor, obstacle, material, shape

\section{INTRODUCTION}

Glasses for Blind and Visually Impaired is a device built to help blind and visually impaired to navigate around diverse range of obstacles. This device consists of two parts. First part is a head mounted sensor assembly whereas the second is a haptic feedback device, worn as a belt. Detection of obstacles is performed by 10 VL53L1X Time of Flight (ToF) sensors [1], whose ranging data is then processed with an on-board ESP-WROOM-32 microcontroller [2] and send via Bluetooth connection to the belt. The belt is equipped with a second ESP-WROOM-32 on-board microcontroller, which interprets the data and presents it to the wearer with 15 vibration motors arranged in a square grid. The glasses are worn on the head, whereas the belt is attached around the stomach area.

The motivation behind this work is the desire for this device to work in as many circumstances as possible. Rapid technological development brings new materials and shapes in our everyday living space. If we add fast lifestyle to the mix, we get the need for a device that can reliably detect different shapes and materials in close to real time.

Performance of the device was already tested on specifically designed polygon with 12 test subjects. The results are written in the next section. Expanding on these results, in this study, we focus on testing and quantifying device's ability to detect different shapes and materials.

\section{POLYGON TEST RESULTS}

Test took place in the main lobby of the Faculty of Electrical Engineering and Computer Science, Maribor. This area was used because it is big and open so the glasses detected the obstacles only. It also provided room lighting conditions and flat surface. Polygon seen on Figure 1 consisted out of 4 obstacles made out of polystyrene foam. They simulated everyday objects such as tables, poles and doors. Fifth obstacle which simulated hanging obstacle was tested individually. Every participant had two attempts. Test focused on the number of detected obstacles, number of steps and time necessary to finish the walk through the polygon [3].

All participants detected the fifth, hanging obstacle, but only one out of 12 participants detected an obstacle which simulated the table. On average $2.8 \pm 0.39$ out of 4 obstacles were detected in the first run and $2.92 \pm 0.29$ out of 4 in 
the second run. Average time required for participants to finish the walk through the polygon was $80 \pm 31.5$ seconds in the first and $56 \pm 18.3$ seconds in the second run. On average, participants made $56.2 \pm 15.7$ steps in the first and $48.3 \pm 10.7$ in the second run [3].

From increased walking speed on the second attempt we can conclude that participant certainty with the device increased over time. This was also reflected in improved obstacle detection. [3]

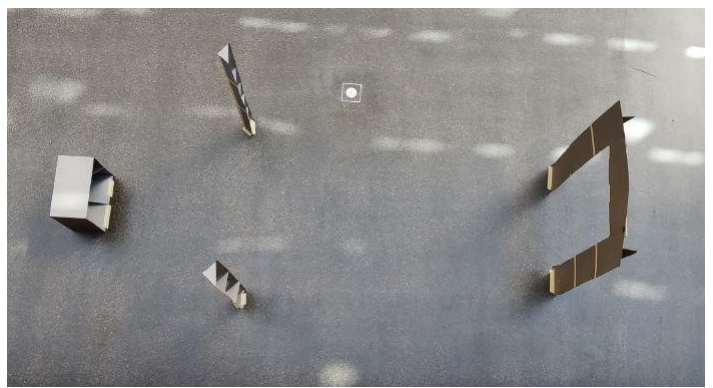

Figure 1: test polygon, set up in the main lobby of the Faculty of Electrical Engineering and Computer Science, Maribor.

\section{DETECTION ZONE}

Detection of obstacles is implemented using ToF sensors VL53L1X. They provide a maximum range of $4 \mathrm{~m}$ and a typical field of view of $27^{\circ}$. These sensors were selected because they are relatively affordable, offer long range detection, are power efficient, support I2C interface and are small enough to enable slim design [1].

Detection zone of the device is constructed by an array of 10 sensors, providing $150^{\circ}$ wide and $50^{\circ}$ tall coverage as seen on Figure 3. Sensors are divided into three groups. First group consists of two sensors which are oriented straight in the direction of view. One sensor is oriented horizontally and the other is oriented 30 degrees below the horizon. Second group consists of 6 sensors, 3 on the left side and 3 on the right side. Left group is vertically tilted for $22^{\circ}$ to the left, whereas right group is vertically tilted for $22^{\circ}$ to the right. The upper two and the lower two sensors in this group are horizontally tilted for $10^{\circ}$ away from the central sensor which is oriented straight into direction of view. Third group consists of 2 sensors which are oriented straight into direction of view and vertically tilted for $44^{\circ}$ [4].

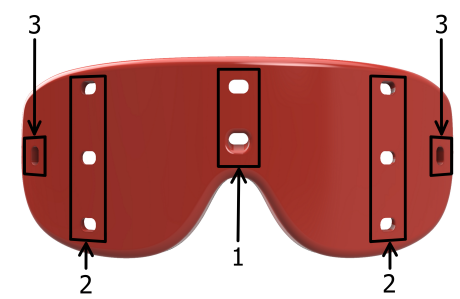

Figure 2: Groups of TOF sensors.
The device was designed in the shape of wearable glasses and 3D printed from Polylactic Acid plastic [4]. This technique was used to achieve special shape which allows sensors to be mounted in a way seen on Figure 3

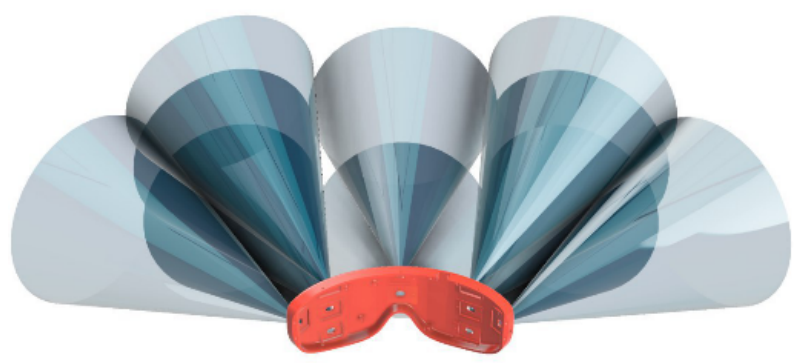

Figure 3: Fields of view for TOF sensors, mounted on the $3 \mathrm{D}$ printed glasses.

\section{OBSTACLE DETECTION}

We designed a special experiment to test how well the glasses detect obstacles of various shapes and materials. Shapes used were circle, triangle and square. Their surface measured $2116 \mathrm{~cm}^{\wedge} 2$. Materials used were grey polystyrene foam, white paper, aluminium foil, glass, polyester, plexiglass, ABS, wood, micro polyester and cotton.

Experiment took place in closed environment under dim lighting conditions. Controlled light conditions for this test are important as they effect sensors performance. Device was mounted on a $176 \mathrm{~cm}$ high stationary wooden stand which pointed directly into the obstacle centre. Obstacles were placed 30,60 and $90 \mathrm{~cm}$ away from the glasses. Quality of detection was determined with data output consisting of 10 integers, ranging from 0 to 4096. Here, value 0 denotes the minimal and value 4096 the maximal distance. In order to increase the accuracy, every distance was calculated by averaging ten measurements.

Shape discrimination ability was assessed by counting the number of sensors that detected the obstacle. Here, we also considered the distance of obstacle from the glasses.

The initial testing session consisted of recognizing different shapes at the distance of $30 \mathrm{~cm}$ (Figure 4). The sensors could not recognize the shape at all. This suggests that the distance is too small for sensors to reach their full potential. 


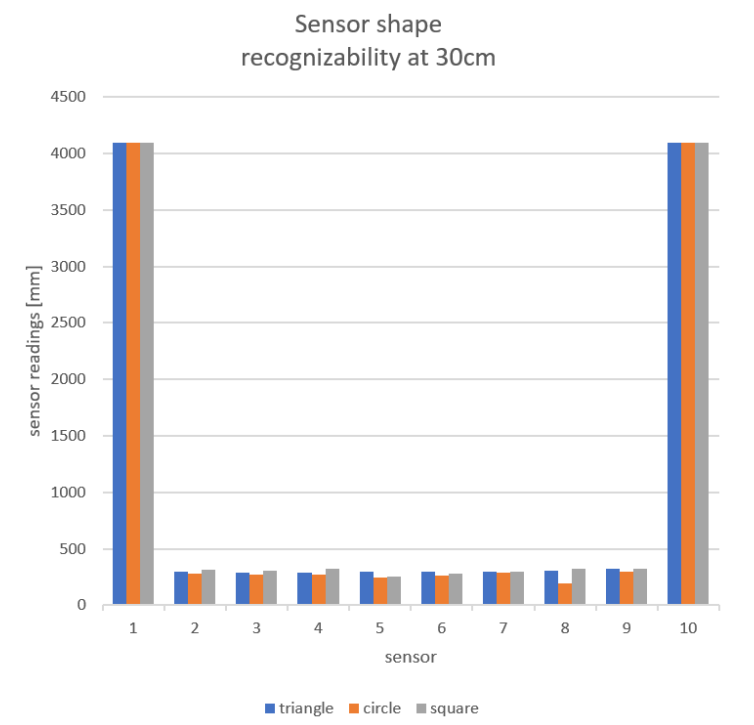

Figure 4: Results of obstacle detection at the distance of $30 \mathrm{~cm}$.

In the next testing session, the obstacles were positioned at the distance of $60 \mathrm{~cm}$ from the sensors (Figure 5). This time the circle and the square were not differentiated, but the triangle was. Sensors 6 and 7 have pointed out a difference.

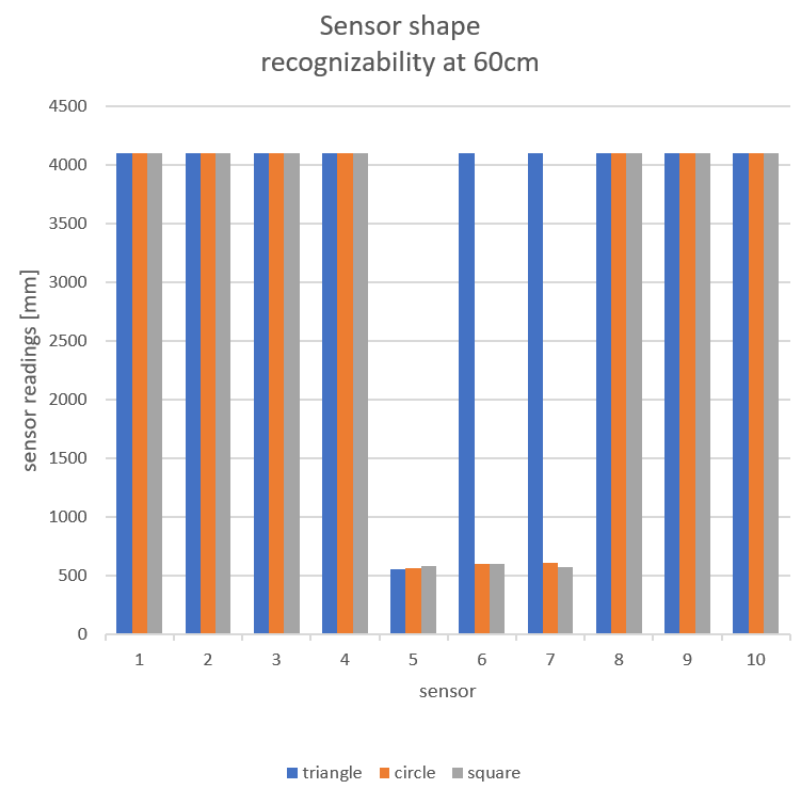

Figure 5: Results of obstacle detection at the distance of $60 \mathrm{~cm}$.

In the third testing session, the obstacles were positioned $90 \mathrm{~cm}$ away from the sensors (Figure 6). In this case, the recognizability was again poor. The distance turned out to be too great, since only one (the middle) sensor detected any of the shapes.

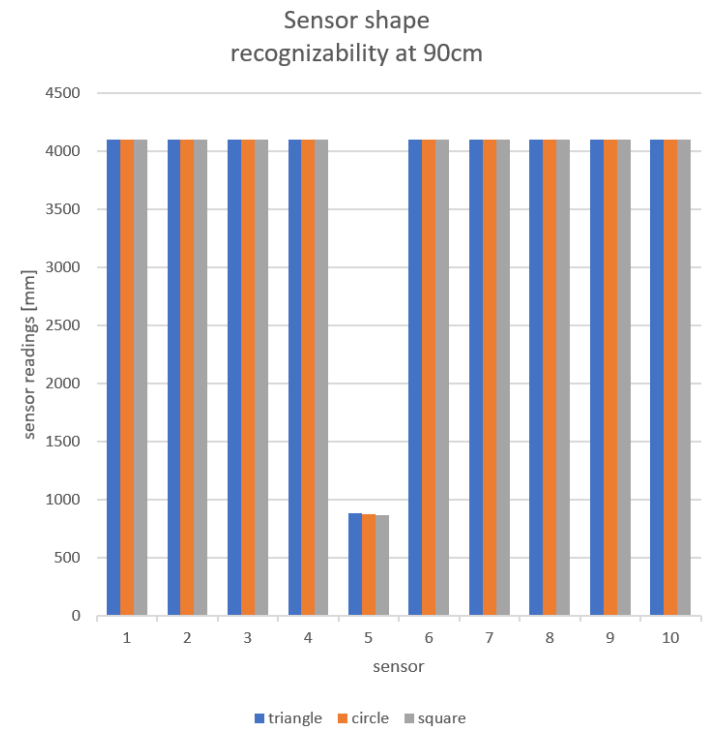

Figure 6: Results of obstacle detection at the distance of $90 \mathrm{~cm}$.

In the last experimental session we tested the detectability of different materials. For this purpose, we put the square obstacle with surface area of $2116 \mathrm{~cm}^{2}$ encased with the chosen material at the maximum detection distance, which was determined by moving the obstacle away from the glasses until it was no longer detected. The results demonstrated (Figure 7) that the aluminium foil has the best detection potential while ABS has the worst. Glass was also tested, but was excluded from the results in Figure 7, because its detection depends on the sensor angle. Under the specific angle glasses could detect it up to the distance of $1.5 \mathrm{~m}$, but in most cases glass wasn't detected. Plexi glass was also interesting because it was consistently detected, but only by one or two sensors, even on a $30 \mathrm{~cm}$ distance.

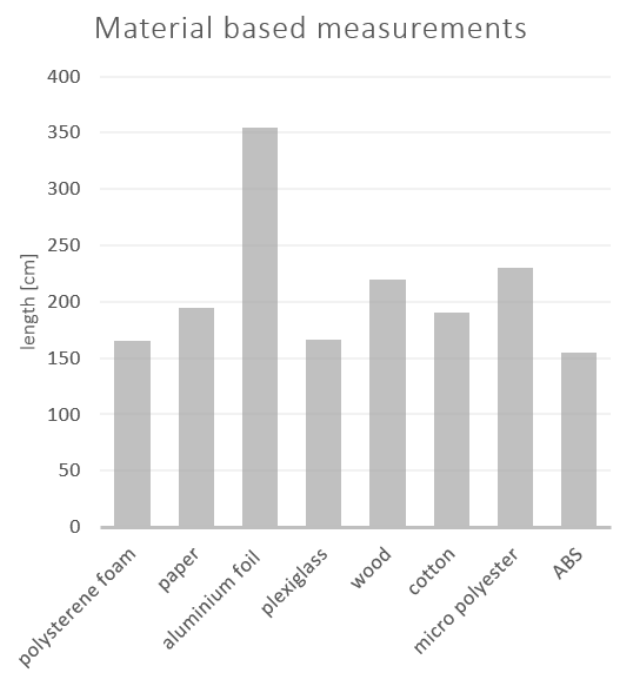

Figure 7: Maximum detection distances for tested materials. 


\section{CONCLUSIONS}

The glasses detected all the obstacles, but the number of sensors detecting the obstacle decreased with the distance. Circle and square were detected better than triangle. This suggests that different shapes trigger different responses of sensors on glasses.

We have also demonstrated that the optimal distance for the sensors to recognize the shapes is somewhere between 30 and $90 \mathrm{~cm}$. At the distance of $60 \mathrm{~cm}$ the triangle was successfully discriminated from circle and rectangle, whereas the latter two were not easy to discriminate. The biggest discriminative power of glasses likely lies at the distances between 30 and $60 \mathrm{~cm}$. However, additional tests are required to analyse the performance at different points in this interval (for example at 40 and $50 \mathrm{~cm}$ ).

The problem with misdetection of more distant obstacles is a consequence of detection principle used in our design. Emitted light cone is $27^{\circ}$ wide and it expands with the distance. As a result, the obstacle takes up smaller part of the cone and that affects its detection. There are two solutions of this problem. The first one is to narrow the emitted light cone width by software or hardware. The second one includes adding the video camera for better object recognition.

Our experiments further showed that some of the materials are poorly detected. For example plexi glass and glass present a substantial detection challenge. On the other hand, aluminium foil, white paper and micro polyester are easily detected. In conclusion, the more reflective the material, the more sensors detect it. Further tests are required to analyse whether or not the problem of glass detection could be addressed by the use of video camera. As an alternative, an ultrasonic sensor could also be used.

At the moment our glasses perform best in open environments while detecting materials which are better at reflecting infrared light. The test justifies that the glasses could be used in everyday environments, because materials tested make up most of potential obstacles, glass and other similar materials being the exception.

Plasticity of the brain allows blind and visually impaired people to have significantly increased perception of touch [4]. Because of that, we would like to additionaly test if they can feel the difference between various shapes.

\section{ACKNOWLEDGMENTS}

We thank prof. dr. Aleš Holobar (University of Maribor) and M.S. Jernej Kranjec (University of Maribor) for their assistance in writing the article and all the professional advises they provided. We also thank Tomaž Ferbežar (Secondary school of electronics and technical gymnasium) and Boris Plut (Secondary school of electronics and technical gymnasium) for their help in the early stages of development.

\section{REFERENCES}

[1] STMicroelectronics https://www.st.com/resource/en/datasheet/vl53l1x.pdf.

[2] Adafruit HUZZAH32 - ESP32 Feather. https://cdn-learn.adafruit.com/downloads/pdf/adafruithuzzah32-esp32-feather.pdf. May 2019.

[3] K. Stopar. Naprava za vizualno-kinestetično navigacijoslepih in slabovidnih. September 2019 (submitted for review).

[4] C. M. Bauer, G. V. Hirsch, L. Zajac, B. Koo, O. Collignon, L. B.

Merabet Multimodal MR-imaging reveals large-scale structural and functional connectivity changes in profound early blindness PLOSjONE. March 22, 2017. 